\title{
CARDINAL FUNCTIONS FOR $k$-SPACES
}

\author{
JAMES R. BOONE, SHELDON W. DAVIS AND GARY GRUENHAGE
}

\begin{abstract}
In this paper, four cardinal functions are defined on the class of $\boldsymbol{k}$-spaces. Some of the relationships between these cardinal functions are studied. Characterizations of various $k$-spaces are presented in terms of the existence of these cardinal functions. A bound for the ordinal invariant $\boldsymbol{\kappa}$ of Arhangelskii and Franklin is established in terms of the tightness of the space. Examples are presented which exhibit the interaction between these cardinal invariants and the ordinal invariants of Arhangelskii and Franklin.
\end{abstract}

1. Introduction. The class of $k$-spaces, introduced by Arens [1], has become a widely used class of topological spaces, both because of their intrinsic interest and because they provide a natural category of spaces in which to study a variety of other topological problems. The $k$-spaces are precisely those spaces which are the quotient spaces of locally compact spaces. In particular, $X$ is a $k$-space provided: $H$ is closed in $X$ if and only if $H \cap K$ is closed in $K$, for each compact set $K$. That is, $X$ has the weak topology induced by the collection of compact subspaces. Franklin [5] initiated the study of a restricted class of $k$-spaces, called sequential spaces. This is the class of $k$-spaces in which the weak topology is induced by the collection of convergent sequences. If $A \subset X$, let $k \operatorname{cl}(A)$ be the set of all points $p$ such that $p \in \operatorname{cl}_{K}(A \cap K)$ for some compact set $K$, and let $s \operatorname{cl}(A)$ be the set of all points $p$ such that there exists a sequence in $A$ that converges to $p$.

Arhangelskii and Franklin [3] introduced the concept of ordinal invariants for topological spaces, which will be called here the compact order and the sequential order of a space. Let $A$ be a subset of a topological space $X$. Let $A^{0}=A$, and for each nonlimit ordinal $\alpha=\beta+1$, let $A^{\alpha}=k \operatorname{cl}\left(A^{\beta}\right)\left[A^{\alpha}=\right.$ $\left.s \operatorname{cl}\left(A^{\beta}\right)\right]$. If $\alpha$ is a limit ordinal, let $A^{\alpha}=\bigcup\left\{A^{\beta}: \beta<\alpha\right\}$. The compact order [sequential order] of $X$ is defined as

$$
\kappa(X)[\sigma(X)]=\inf \left\{\alpha: A^{\alpha}=\operatorname{cl}(A), \text { for all } A \subset X\right\} .
$$

These infima do not exist for all topological spaces as the following theorems and Example 3.1 indicate.

THEOREM 1.1 [3]. $X$ is a $k$-space if and only if $\kappa(X)$ exists.

Presented to the Society, May 5, 1977; received by the editors May 2, 1977 and, in revised form, July 18, 1977.

AMS (MOS) subject classifications (1970). Primary 54A25; Secondary 54F65, 54D50, 54D55. $K e y$ words and phrases. Cardinal function, ordinal invariant, $\boldsymbol{k}$-space. 
TheOREM 1.2 [3]. $X$ is a sequential space if and only if $\sigma(X)$ exists. In this case $\sigma(X) \leqslant \omega_{1}$.

The primary purpose of this paper is to introduce cardinal invariants for the class of $k$-spaces and give a bound for the ordinal invariant $\kappa$ in Theorem 1.1. We show that $\kappa(X) \leqslant t(X)^{+}$, where $t(X)$ is the tightness of $X$. Note that this is a generalization of the sequential case, because if $X$ is sequential, then $t(X)=\omega_{0}$ and $\kappa(X) \leqslant \sigma(X) \leqslant \omega_{1}$.

\section{Definitions and a characterization of $k$-spaces.}

DefinItION 2.1. Let $A$ be a subset of a space $X$. The pointwise $k$-cardinal of $A, P K(A)$, is the least $\beta$ such that for every $p \in \operatorname{cl}(A)$ there exists $\alpha$ with $p \in A^{\alpha}$ and $\operatorname{card}(\alpha) \leqslant \beta$.

Definition 2.2. Let $A$ be a subset of a space $X$. The $k$-cardinal, $K(A)$, of $A$ is the cardinality of the least ordinal $\alpha$ such that $\operatorname{cl}(A)=A^{\alpha}$.

We make the following observations:

(a) where defined, $P K(A)=\sup \left\{\operatorname{card}(\alpha): A^{\alpha+1}-A^{\alpha} \neq \varnothing\right\}$.

(b) where defined, $K(A)=\operatorname{card}\left(\sup \left\{\alpha: A^{\alpha+1}-A^{\alpha} \neq \varnothing\right\}\right)$.

The pointwise sequential cardinal, $P S(A)$, and the sequential cardinal, $S(A)$, of $A$ are defined in the same manner as above. The analogues of (a) and (b) hold for these functions. Also, just as $\kappa(X) \leqslant \sigma(X)$, so too $K(X) \leqslant S(X)$ and $P K(X) \leqslant P S(X)$. The relationship between these cardinal numbers, when they exist, for a set $A$ is indicated in the following.

Proposition 2.3. If $A$ is a subset of a space $X$, then $P K(A) \leqslant K(A) \leqslant$ $P K(A)^{+}$and $P S(A) \leqslant S(A) \leqslant P S(A)^{+}$.

Definition 2.4. Let $X$ be a topological space. The pointwise $k$-cardinal of $X$, $P K(X)$, and the $k$-cardinal of $X, K(X)$, are defined as:

$$
P K(X)=\sup \{P K(A): A \subset X\} \quad \text { and } K(X)=\sup \{K(A): A \subset X\} \text {. }
$$

$(P S(X)$ and $S(X)$ are defined similarly.)

Note that, when $K(X)$ is defined, we have $K(X)=\operatorname{card}(\kappa(X))$. Similarly, when $S(X)$ is defined, $S(X)=\operatorname{card}(\sigma(X))$.

COROLlary 2.5. If $X$ is a topological space, then $P K(X) \leqslant K(X) \leqslant$ $P K(X)^{+}$and $P S(X) \leqslant S(X) \leqslant P S(X)^{+}$.

Let $t(X)$ be the tightness of $X$, as defined by Arhangelskii [2]. In short, if $X$ has tightness $t(X)$ and $p \in \operatorname{cl}(A)$ then there exists a subset $B \subset A$ such that $p \in \operatorname{cl}(B)$ and $\operatorname{card}(B) \leqslant t(X)$. It is the tightness of a space that yields a bound on the ordinal invariant $\kappa$.

THEOREM 2.6. $X$ is a $k$-space if and only if $P K(X)$ exists. In this case $P K(X) \leqslant t(X)$.

Proof. The first part is easy, and we omit the proof. Suppose $X$ is a $k$-space and $P K(X)>t(X)$. Then there is some $A \subset X$ such that $P K(A)>$ $t(X)$. Let $\eta=t(X)^{+}$. There is a point $x \in A^{\eta+1}-A^{\eta}$, and so there is a 
compact set $K$ such that $x \in \operatorname{cl}_{K}\left(K \cap A^{\eta}\right)-\left(K \cap A^{\eta}\right)$. Hence there is a set $C \subset K \cap A^{\eta}$ such that $\operatorname{card}(C) \leqslant t(X)$ and $x \in \operatorname{cl}(C)$. For each $y \in C$, there is some $\delta<\eta$ such that $y \in A^{\delta}$. Since $\eta=t(X)^{+}$is a regular cardinal, there exists $\gamma<\eta$ such that $C \subset A^{\gamma}$. Thus $x \in \operatorname{cl}_{K}(C) \subset \operatorname{cl}_{K}\left(K \cap A^{\gamma}\right) \subset$ $A^{\gamma+1}$. Thus $x \in K \cap A^{\eta}$, which is a contradiction.

Since the proof would be the same and Arhangelskii's assertion that every sequential space has countable tightness, has been proven in [4], the following is true.

THEOREM 2.7. $X$ is a sequential space if and only if $P S(X)$ exists. In this case, $P S(X) \leqslant \aleph_{0}$.

From the previous theorems and the boundedness property in Corollary 2.5 , the following are valid.

THEOREM 2.8. $X$ is a $k$-space if and only if $K(X)$ exists. In this case $K(X) \leqslant t(X)^{+}$.

THEOREM 2.9. $X$ is a sequential space if and only if $S(X)$ exists. In this case $S(X) \leqslant \boldsymbol{x}_{1}$.

From the definitions we also have the following.

THEOREM 2.10. (a) $X$ is a $k^{\prime}$-space if and only if $P K(X)=K(X)=1$.

(b) $X$ is a Fréchet space if and only if $P S(X)=S(X)=1$.

As a direct consequence of Theorem 2.8 we can now supply a bound for the ordinal invariant $\kappa$ for each space $X$.

Corollary 2.11. $X$ is a $k$-space if and only if $\kappa(X)$ exists. In this case, $\kappa(X) \leqslant t(X)^{+} ;$in fact,

$K(X)=P K(X)$ implies $\kappa(K) \leqslant t(X)$, and

$K(X)=P K(X)^{+}$implies $\kappa(X)=t(X)^{+}$.

3. Examples. The examples presented here are intended to exhibit some of the relationships between the various cardinal and ordinal functions considered in this paper. Example 3.1 is a unification of a variety of treatments of Arens' space. Example 3.2 is the space $S_{\omega}$ of [3]. This example displays the difference between the cardinal functions $P K$ and $K$.

Example 3.1. Generalized Arens Space. A space $S$ is defined for which $\kappa(S)=\sigma(S)=\omega_{0}, \quad P K(S)=P S(S)=\aleph_{0}=K(S)=S(S)$ and which contains for each $n \in N$, a closed subspace $S_{n}$ such that $\kappa\left(S_{n}\right)=\sigma\left(S_{n}\right)=n$ and $\operatorname{PK}\left(S_{n}\right)=\operatorname{PS}\left(S_{n}\right)=n=K\left(S_{n}\right)=S\left(S_{n}\right)$. $S$ also contains a subspace $A$ which is not a $k$-space. That is, none of the ordinal or cardinal functions are defined for A.

$S$ will be the quotient space of the countable disjoint union of the convergent sequences, $T=\{0\} \cup\{1 / n: n \in N\}$. Let $F_{m}=N^{m}$ for each $m \in N$, and let $F=\cup_{n \in N} F_{n} \cup\{0\}$. For each $f \in F$, say $f=\left(m_{1}\right.$, $\left.m_{2}, \ldots, m_{k}\right)$, let $T_{f}=\left\{\left(m_{1}, m_{2}, \ldots, m_{k}, x\right): x \in T\right\}$. Let $X$ be the disjoint 
topological union of the spaces $T_{f}, f \in F$, together with $T . X$ is a countable, locally compact metric space. Let $S$ be the set of all sequences $s=\left(s_{1}\right.$, $s_{2}, \ldots$ ) of length $\omega$ such that for some $n_{s} \in N$,

(a) $n \geqslant n_{s}$ implies $s_{n}=0$, and

(b) $n<n_{s}$ implies $s_{n}=1 / k$ for some $k \in N$.

Let $S$ have the quotient topology induced by the mapping $\phi: X \rightarrow S$ where

$$
\phi\left(m_{1}, \ldots, m_{k}, x\right)=\left(1 / m_{1}, 1 / m_{2}, \ldots, 1 / m_{k}, x, 0,0, \ldots\right),
$$

and $\phi(x)=(x, 0,0, \ldots)$. That is, $\phi$ attaches the point $\left(m_{1}, \ldots, m_{k}, 0\right)$ to the point $\left(m_{1}, \ldots, m_{k-1}, 1 / m_{k}\right)$ and $\left(m_{1}, 0\right)$ to $1 / m_{1} \in T$.

$S$ is a sequential space and contains, as a closed subspace, each of the $S_{n}$ which are inductively constructed in [3]. In particular, $S_{n}=\left\{s \in S: s_{k}=0\right.$ for each $k>n\}$, for each $n \in N$. For each $n \in N, P K\left(S_{n}\right)=P S\left(S_{n}\right)=n=$ $K\left(S_{n}\right)=S\left(S_{n}\right)$ and $\kappa\left(S_{n}\right)=\sigma\left(S_{n}\right)=n$. Also, $P K(S)=P S(S)=\aleph_{0}=$ $K(S)=S(S)$ and $\kappa(S)=\sigma(S)=\omega_{0}$.

The most frequently used subspace of $S$ is $S_{2}=\{(0,0)\} \cup\{(1 / m, 0)$ : $m \in N\} \cup\{(1 / m, 1 / n): m, n \in N\}$ as it contains the space of Arens [1], $A=\{(0,0)\} \cup\{(1 / m, 1 / n): m, n \in N\} . A$ is not a $k$-space, since $(0,0) \in$ $\operatorname{cl}(\{(1 / m, 1 / n): m, n \in N\})$, but $\{(1 / m, 1 / m): m, n \in N\} \cap K$ is closed in $K$, for each compact set $K \subset A$. Accordingly, none of the cardinal or ordinal functions discussed in this paper are defined on $A$.

EXAMPLE 3.2. The Space $S_{\omega}$ of Arhangelskii and Franklin [3]. There is a countable sequential space $S_{\omega}$ for which $P K\left(S_{\omega}\right)=\aleph_{0}$ and $K\left(S_{\omega}\right)=\aleph_{1}$.

$S_{\omega}$ is a countable sequential space with $\kappa\left(S_{\omega}\right)=\sigma\left(S_{\omega}\right)=\omega_{1}$. For each $\alpha<\omega_{1}$, there is a space $K_{\alpha}$ with $\kappa\left(K_{\alpha}\right)=\sigma\left(K_{\alpha}\right)=\alpha$. For each $\alpha<\omega_{1}$, $P K\left(K_{\alpha}\right)=P S\left(K_{\alpha}\right)=K\left(K_{\alpha}\right)=S\left(K_{\alpha}\right) \leqslant \aleph_{0}$ and a homeomorphic copy of $K_{\alpha}$ is contained in $S_{\omega}$ for each $\alpha<\omega_{1}$. Let

$$
K=\bigcup\left\{K_{\beta}: \beta<\omega_{1} \text { and } \beta \text { is a nonlimit ordinal }\right\} \subset S_{\omega} \text {. }
$$

$P K(K)=\aleph_{0}$, but $K(K)=\aleph_{1}$, because $\omega_{1}$ is the least ordinal such that $\operatorname{cl}(K)=K^{\omega_{1}}$. Since $K \subset S_{\omega}, K\left(S_{\omega}\right)=S\left(S_{\omega}\right)=\aleph_{1} . P K\left(S_{\omega}\right)=P S\left(S_{\omega}\right)=\aleph_{0}$. (The space $X$ consisting of the disjoint topological union of the spaces $K_{\alpha}$, $\alpha<\omega_{1}$, also serves as a model for $P K(X)=\aleph_{0}$ and $K(X)=\aleph_{1}$. However, $X$ is uncountable.)

\section{REFERENCES}

1. R. Arens, Note on convergence in topology, Math. Mag. 23 (1950), 299-234.

2. A. Arhangel'skii, The power of bicompacta with the first axiom of countability, Soviet Math. Dokl. 10 (1969), 951-955.

3. A. Arhangel'skii and S. P. Franklin, Ordinal invariants for topological spaces, Michigan Math. J. 15 (1968), 313-320.

4. S. W. Davis, A study of certain classes of isocompact spaces and of the relationships among them, Dissertation, Ohio University, 1976.

5. S. P. Franklin, Spaces in which sequences suffice, Fund. Math. 57 (1965), 107-115.

Department of Mathematics, Texas A\&M University, College Station, Texas 77843 (Current address of J. R. Boone)

Department of Mathematics, Auburn University, Auburn, Alabama 36830 (Current address of S. W. Davis and Gary Gruenhage) 